\title{
Towards a better adaptability of hand prostheses to improve its acceptance by amputees
}

\begin{abstract}
Improving the functionality of hand prostheses using non-invasive techniques controlled by the user intention continues being a focus of interest. Although myoelectric control has been widely studied, there are still lacks in the functionality and its applicability in real-time. Additionally, the application of myoelectric prostheses on amputees is still a challenge due to different factors related to non-clinical environments, as well as variations inherent in these subjects. This opinion includes a brief perspective of the trends to be addressed, in order to improve the functionality of these prosthetic devices.
\end{abstract}

Keywords: myoelectric signals, hand prosthesis, pattern recognition
Volume 4 Issue 2 - 2018

\author{
John Jairo Villarejo Mayor,' Denis Delisle \\ Rodriguez ${ }^{2,3}$ \\ 'Federal University of Parana, Brazil \\ ${ }^{2}$ Federal University of Espirito Santo, Brazil \\ ${ }^{3}$ Center of Medical Biophysics, Cuba
}

\begin{abstract}
Correspondence: John Jairo Villarejo Mayor, Ph D in Electrical Engineering, Postdoctoral Research Fellow

Federal University of Parana, Coracao de Maria Street, 92, Curitiba - Parana, Brazil,Tel +55 4l 99873084I,

Email jvimayor@gmail.com
\end{abstract}

Received: March 08, 2018 | Published: April 18, 2018

\section{Abbreviations: sMES: superficial myoelectric signals \\ Introduction}

The design and control of versatile upper-limb prostheses is a very challenging task. While many breakthroughs have been made over the last several decades, the difference in performance and quality between human hands and artificial hands is quite substantial. Robotic hands may present several degrees-of-freedom distributed among several kinematic chains and fingers. The complexity of the mechanical design is necessary to adapt hands for executing different tasks on unstructured environments. Although it has been acknowledged that a prosthetic limb does not provide a full functionality for amputated human limb, in the last decade there have been many advances to improve the upper-limb prosthetic restoration. ${ }^{1-4}$ However, the current prosthetic solutions give a reduced control capability due to limitations of interfaces adopted for controlling the prosthesis. ${ }^{5}$ One of the major problems is the acceptance of prostheses by amputees, taking into account their facilities and comfort, exterior appearance, but most of all, its functionality. ${ }^{6}$ Current researches and development of upper-limb prostheses continue to be held to mimic the human hand in terms of dexterity and adaptive capacity. The literature shows that movement intention should be recognized and selected by the user, while the grasp and movement executions should be performed automatically into a low-level control system, making sure to hold objects and avoid slippage. ${ }^{7}$

There is an increasingly interest to provide more functionality on the current prostheses for improving the acceptation of amputees, ${ }^{6}$ which are addressed to be able to identify dexterous tasks and different precision grasps to increase the skills on task of daily living. ${ }^{5,8,9}$ The myoelectric controlled prosthetic hand are able to recognize the user intention from patterns of different daily tasks to perform movements in a more natural way. These mechanisms should include individual control of fingers and tasks of greater skill for the grip of different objects, which represents a great challenge in the design of hand prostheses. However, the superficial myoelectric signals (sMES) generated by movements of individual fingers are weaker than those generated by movements of the wrist. Further, the literature has reported a non-linear relationship between strength and muscle electrical activity in conditions of low level contractions. ${ }^{10}$ Non-linear techniques have been used to model weak sMES instead of high forces, in order to develop control systems of high level providing a more natural human-machine interaction. ${ }^{7,11,12}$ In addition, the use of a reduce number of electrodes has been proposed as a promising improvement of the accuracy of these systems, avoiding interferences, training and decreasing computational cost for real time implementations. ${ }^{11-13}$ Currently, one of the most important focus of researches is to develop control strategies for real-time using machine learning to analyze the sMES during the control of dexterous prosthetic hands, applied in practice with amputees. The machine learning is trained by muscle patterns in an initial stage of the designing through a pattern recognition system. In order to achieve this goal, these patterns learned are required to be stable. However, these patterns are not static, they are continuously modified as the signal may be interfered by shift, fatigue and user-unfriendly of retraining, ${ }^{14}$ as well as variations of patterns by the constant use of the prosthesis. Adaptive learning algorithms may be suitable to retrain the classification model, incorporating new data to the previous model to improve the performance. Therefore, a long training period to obtain a finer control is sought. Moreover, clinical parameters related to amputees have a relationship with the performance and the number of independent movements controlled by the amputee. ${ }^{15}$ The selection of a suitable hand gestures for a reliable myoelectric control is an interesting focus of research. ${ }^{16}$ The learning ability (adaptation) of the individual should be taken into account in the rehabilitation process. Therefore, it is important to consider the inclusion of adaptive methods and constant learning in the pattern recognition systems, in order to improve the functionality and adaptability of the prosthesis.

\section{Conclusion}

In conclusion, the design of myoelectric prostheses should be aim to develop systems that are more functional for amputees, considering that the amputee can improve their abilities to distinguish movements from the continuous use of the prosthesis. The adaptability of these 
systems should be considered as an interesting focus of study, so that prostheses stop being complex technology devices, expensive, but in many cases, it is rejected by users.

\section{Acknowledgements}

None.

\section{Conflict of interest}

Authors declare that there is no conflict of interest.

\section{References}

1. Burck J, Bigelow J, Harshbarger S. Revolutionizing Prosthetics: Systems Engineering Challenges and Opportunities. Johns Hopkins APL Tech Dig. 2011;30(3):186-197.

2. Cipriani C, Controzzi M, Carrozza MC. The Smart Hand transradial prosthesis. J Neuroeng Rehabil. 2011;8(1):29.

3. Losier Y, Clawson A, Wilson A, et al. An Overview of the UNB Hand System. In Proceedings of the 2011 MyoElectric Controls/Powered Prosthetics Symposium Fredericton. 2011:2-5.

4. Matrone GC, Cipriani C, Carrozza MC, et al. Real-time myoelectric control of a multi-fingered hand prosthesis using Principal Components Analysis. J Neuroeng Rehabil. 2012;9(1):40.

5. Cordella F, Ciancio AL, Sacchetti R, et al. Literature Review on Needs of Upper Limb Prosthesis Users. Front Neurosci. 2016;10:209.

6. Peerdeman B1, Boere D, Witteveen H, et al. Myoelectric forearm prostheses: State of the art from a user-centered perspective. J Rehabil Res Dev. 2011;48(6):719-37.
7. Villarejo Mayor JJ, Mamede Cota R, Frizera Neto A, et al. Dexterous Hand Gestures Recognition Based on Low-Density sEMG Signals for Upper-Limb Forearm Amputees. Res Biomed Eng. 2017;33(3):202-217.

8. Phinyomark A, Quaine F, Charbonnier S, et al. EMG feature evaluation for improving myoelectric pattern recognition robustness. Expert Syst Appl. 2013;40(12):4832-4840.

9. Geethanjali P. Myoelectric control of prosthetic hands: state-of-the-art review. Med Devices Evid Res. 2016;9:247-255.

10. Naik GR, Kumar DK. Identification of hand and finger movements using multi run ICA of surface electromyogram. J Med Syst. 2012;36(2):841851.

11. Phinyomark A, Phukpattaranont $\mathrm{P}$, Limsakul C. Fractal analysis features for weak and single-channel upper-limb EMG signals. Expert Syst Appl. 2012;39(12):11156-11163.

12. Kumar DK, Poosapadi Arjunan S, Singh VP. Towards identification of finger flexions using single channel surface electromyography - able bodied and amputee subjects. J Neuroeng Rehabil. 2013;10(1):50.

13. Mesa I, Rubio A, Tubia I, et al. Channel and feature selection for a surface electromyographic pattern recognition task. Expert Syst Appl. 2014;41(11):5190-5200.

14. Zhang Y, Wang Z, Zhang Z. Comparison of Online Adaptive Learning Algorithms for Myoelectric Hand Control. 2016;69-75.

15. Atzori M, Gijsberts A, Castellini C, et al. Effect of clinical parameters on the control of myoelectric robotic prosthetic hands. J Rehabil Res Dev. 2016;53(3):345-58

16. Castro MCF, Arjunan SP, Kumar DK. Selection of suitable hand gestures for reliable myoelectric human computer interface. Biomed Eng Online. 2015;14(1):1-11 